\title{
GMR
}

\section{Serum matrix metalloproteinases-3 levels in patients with ankylosing spondylitis}

\author{
J.W. Gao', K.F. Zhang ${ }^{1}$, J.S. Lu ${ }^{1}$ and T. Su ${ }^{2}$ \\ ${ }^{1}$ Department of Spine Surgery, the First People's Hospital of Jining City, \\ Jining, China \\ 2Department of Pediatric Surgery, the First People's Hospital of Jining City, \\ Jining, China \\ Corresponding author: J.W. Gao \\ E-mail: gaojianwen723@126.com
}

Genet. Mol. Res. 14 (4): 17068-17078 (2015)

Received August 9, 2015

Accepted October 25, 2015

Published December 16, 2015

DOI http://dx.doi.org/10.4238/2015.December.16.7

ABSTRACT. Cumulated evidence indicates that matrix metalloproteinase-3 (MMP-3) is significantly involved in cancer progression. Recent studies yielded conflicting results regarding the association between serum MMP3 and ankylosing spondylitis (AS). To clarify this correlation, we performed a meta-analysis. Potential relevant studies were identified by searching the following databases: PubMed, Embase, CINAHL, Science Citation Index database, the Cochrane Library, Current Contents Index, Chinese Biomedical, the Chinese Journal Full-Text, and the Weipu Journal. Data from eligible studies were extracted and included into the meta-analysis using a random-effect model. Standardized mean differences (SMDs) and $95 \%$ confidence intervals (Cls) were used to assess the association between serum MMP-3 levels and AS. Thirteen case-control studies, including 707 AS cases and 442 healthy controls, were selected for the meta-analysis. The results indicate a significantly higher serum MMP3 level in patients with AS than that in the controls (cases vs controls: $\mathrm{SMD}=1.31,95 \% \mathrm{Cl}=0.84-1.78, \mathrm{P}<0.001)$. Ethnicity-subgroup analysis indicated a higher MMP-3 level in Asian and Caucasian patients with AS (all $P<0.05$ ). This meta-analysis indicates that increased serum MMP-3 
level correlates with the development of AS, suggesting that MMP-3 may present a clinical value in reflecting the progression of AS. Further larger sample size studies are warranted.

Key words: Ankylosing spondylitis; Matrix metalloproteinases-3; Meta-analysis; Serum level

\section{INTRODUCTION}

Ankylosing spondylitis (AS) is a type of inflammatory arthritis, which belongs to the spondyloarthritis family that includes reactive arthritis, psoriatic arthritis, undifferentiated spondyloarthritis, and entero-associated arthritis (Bakland and Nossent, 2013). Involving the axial skeleton, AS is also considered a progressive and chronic inflammatory rheumatic disorder, which is frequently related to the presence of extra-articular manifestations and involves peripheral joints (Kassimos et al., 2014). Though the prevalence of AS is difficult to determine, it varies from country to country with a global prevalence between 0 and 1.4\% (Lee et al., 2013). In Asia, AS is one of the three most common systemic inflammatory diseases associated with uveitis (Zhang et al., 2014). Generally, this disease results in harming spinal mobility and physical function, and might lead to serious impairments of structure and function, which eventually have a negative influence on the quality of life of patients (Dean et al., 2014). Additionally, the standardized ratio of deaths in patients with AS is between 1.5 and 1.9, which is higher than anticipated (Halvorsen et al., 2013). The pathogenesis and etiology of AS remain unclear. However, the current hypothesis involves immune mediation, including several cytokines, such as interaction between $\mathrm{T}$ cell response, environmental factors, tumor necrosis factor (TNF), bacterial antigens, and genetic factors as its major mechanism (Wei et al., 2011). Coincidently, the Bath AS disease activity index score showed that there is a correlation between serum matrix metalloproteinase-3 (MMP-3) levels and disease activity. Moreover, MMP-3 represents an independent predictive value of structural impairment progression in patients with AS (Wagner et al., 2012).

MMP-3, consisting of a signal peptide, is widely known as a member of the MMP family, composed of zinc-dependent endopeptidases necessary for normal biological functions, and plays a crucial role in morphogenesis, wound healing, tissue repair, and remodeling in response to injury, and in the development of various diseases, including arthritis, cancer, and chronic tissue ulcers (Visse and Nagase, 2003; Nagase et al., 2006). MMP-3, largely generated by connective tissue cells, is capable of synergistically degrading the main components of the extracellular matrix (ECM) as well as proteoglycan, laminin, fibronectin, and type IV collagen (Jin et al., 2005). In normal tissues, MMP-3 expression is usually relatively low, while its expression changes during tumorigenesis, where remodeling of the ECM is needed (Johansson et al., 2000). Recently, the connection between serum levels of MMP-3 and the pathogenesis of AS garnered the attention (Wendling et al., 2008; Dhir et al., 2013). MMP-3 is known to be an important protease in cartilage damage and joint destruction because it can cleave a variety of ECM proteins (Sun et al., 2014). In addition, MMP-3 is generated in the joint in reaction to local interleukin-6 (IL-6), tumor necrosis factor- $\alpha$ (TNF $\alpha$ ), and interleukin-1 (IL-1) and is considered as a marker of synovial inflammation (Ribbens et al., 2000). AS manifests as a chronic inflammation as evidenced by an increase in pro-inflammatory cytokines such as TNFa and IL-6 (Dhir et al., 2013). Thus, one can speculate that serum MMP-3 levels might correlate with the occurrence of AS. Several epidemiological studies described the implication of 
serum MMP-3 levels in the development and progression of AS, implying that detection of serum MMP-3 levels may be clinically utilized in the evaluation of disease activity in AS (Wendling et al., 2008; Hou et al., 2014). However, other related documents indicated no correlation between serum MMP-3 levels and AS pathogenesis (Lin et al., 2008; Soliman et al., 2012). Therefore, the present meta-analysis aggregated previous findings from relevant studies in an attempt to systematically assess the relationship between serum MMP-3 levels and the etiology of AS.

\section{MATERIAL AND METHODS}

\section{Literature search}

The following computerized bibliographic databases were used to identify relevant articles related to the association of serum MMP-3 concentration and AS susceptibility without restrictions with respect to language or data collection: PubMed, Embase, CINAHL, Science Citation Index, the Cochrane Library, Current Contents Index, Chinese Biomedical, the Chinese Journal FullText, and the Weipu Journal. ("Spondylitis, Ankylosing" or "ankylosing spondylitis" or "Bechterew's disease" or "ankylosing spondyloarthritis" or "rheumatoid spondylitis" or "Marie-Strümpell disease" or "ankylosing spondylitis" or "Von Bechterew's disease") and ("Matrix Metalloproteinase 3" or "Matrix Metalloproteinase-3" or "MMP-3" or "MMP 3" or "MMP3" or "Transin" or "Stromelysin" or "Stromelysin 1" or "MMP-3 Metalloproteinase" or "MMP 3 Metalloproteinase") were entered in the databases searches as medical subject heading terms and text words with a highly sensitive search strategy. A manual search was performed to screen other eligible studies.

\section{Study selection}

After reading the abstract, full papers were retrieved and assessed for their suitability with the following inclusion criteria: 1) only case-control studies conducted within a human population to examine the association between serum MMP-3 concentration and AS susceptibility were incorporated; 2) patients with AS should be confirmed by the modified New York criteria (van der Linden et al., 1984); 3) the article must be published in a peer-reviewed journal and provide original data; 4) supply sufficient information on the serum MMP-3 concentration in the cases and controls. The major exclusion criteria were: 1) did not satisfy the inclusion criteria designed in the current study; 2) abstracts, reviews, case report, letters, meta-analysis, or proceedings; 3) duplication publications or studies with overlapping data; 4) subgroup analysis of the included trials.

\section{Data extraction and quality assessment}

We used a standard reporting form to extract data from each included study and the following descriptive information were collected: surname and initials of the first author, year of submission, country, racial descent, study design, number of cases and controls, demographic variables, MMP-3 detection method, MMP-3 levels in the cases and the controls, and confirmation of diagnosis. Two reviewers independently assessed the methodological quality of the included trials using the Newcastle-Ottawa Scale (NOS) criteria to ensure consistency in reviewing and reporting results. Three aspects were considered in the NOS criteria: 1) subject selection: $0-4 ; 2$ ) comparability of subjects: $0-2 ; 3$ ) clinical outcome: $0-3$. The range of NOS scores is from 0 to 9 and 
a score $\geq 7$ means a good quality. Disagreement on the inclusion of a single study was settled by discussion or a third investigator was consulted.

\section{Statistical analysis}

The association between serum MMP-3 level and AS susceptibility was estimated by the standardized mean difference (SMDs) and 95\% confidence interval $(95 \% \mathrm{Cl})$. We used Cochran's Q-statistic ( $P<0.05$ was considered significant) and $l^{2}$ tests to quantify heterogeneity among studies (Zintzaras and loannidis, 2005a). In order to calculate the pool SMDs, fixed/random effect models were used. The random effects model was applied for the evidence of significant heterogeneity $(P<0.05$ or $l^{2}$ test exhibited $>50 \%$ ), whereas SMDs were pooled based on the fixed-effects model (Higgins and Thompson, 2002; Zintzaras and loannidis, 2005b). Meanwhile, if there was significant heterogeneity, a subgroup analysis was performed to find potential explanatory variables for the differences in serum MMP-3 levels between AS cases and the controls. In addition, we employed a sensitivity analysis to evaluate whether one single study had the weight to impact on the overall estimate. Furthermore, the effect of publication bias was detected by Egger's linear regression test $(P<0.05$ was considered significant), which can be used to evaluate the funnel plot asymmetry that reveals possible publication bias (Song and Gilbody, 1998; Peters et al., 2006). Statistical analyses were conducted with the STATA statistical software (Version 12.0, Stata Corporation, College Station, TX, USA).

\section{RESULTS}

\section{Selection of eligible studies}

Figure 1 shows the flow chart of identified publications and the main reasons for exclusion. First, 98 potential articles were selected from the electronic databases. One was obtained by manual searching. Of the 99 articles, 2 studies were duplicates and, thus, removed. After screening of the title and abstract, 44 irrelevant studies were subsequently excluded. Finally, 38 studies were excluded after more detailed full text assessment and 15 studies were selected for the qualitative analysis. Two additional studies were removed after further assessment. Finally, 13 case-control studies were analyzed in the current meta-analysis (Yang et al., 2004b; Chen et al., 2006; de Seny et al., 2008; Lin et al., 2008; Sun and Yang, 2008; Wendling et al., 2008; Wang et al., 2010; Shen et al., 2011; Ma and Yang, 2012; Soliman et al., 2012; Dhir et al., 2013; Sha and Lei, 2013; Hou et al., 2014).

\section{Demographic variables}

The 13 included studies consisted of 707 AS cases and 442 healthy controls with a sample size ranging from 40 to 270 , and the sample size of $>100$ was considered as a large sample size. Two studies analyzed data obtained with Caucasian subjects, one study was performed with African subjects and the remaining 10 studies were conducted with Asian subjects. In four studies, the gender information was lost in the controls and four studies failed to provide the age information for the controls. The detection of serum MMP-3 levels was performed by using enzyme linked immunosorbent assay (ELISA) in the 13 included studies. The MMP-3 levels in the cases and controls were expressed as mean \pm SD. The baseline characteristics and serum MMP-3 levels of the individual studies are summarized in Table 1. 


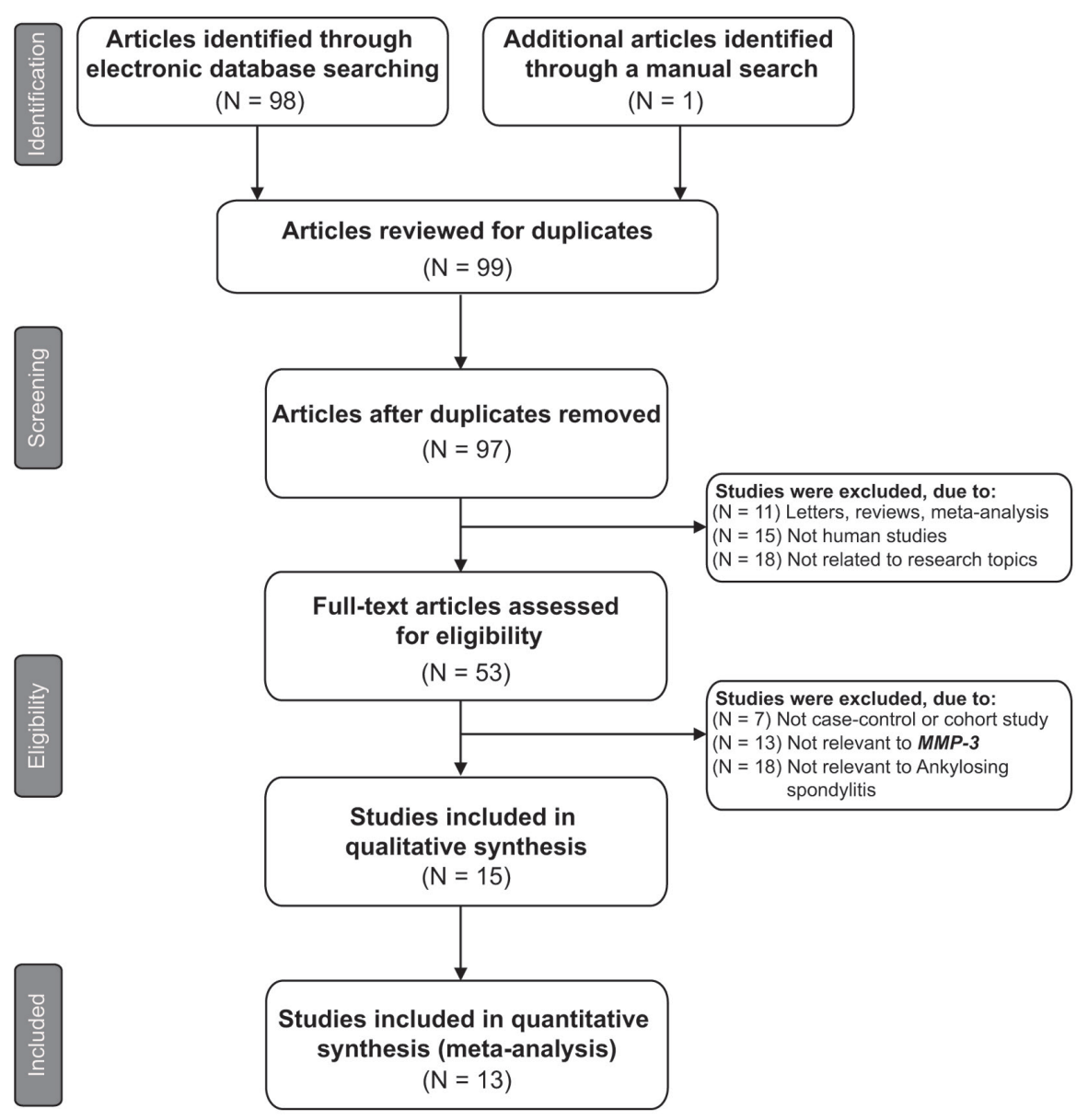

Figure 1. Flow chart of the study selection procedure. Thirteen case-control studies were included in this meta-analysis.

Table 1. Characteristics of included studies focused on MMP-3 serum level.

\begin{tabular}{|c|c|c|c|c|c|c|c|c|c|c|}
\hline \multirow[t]{2}{*}{ First author } & \multirow[t]{2}{*}{ Ethnicity } & \multirow[t]{2}{*}{ Sample } & \multicolumn{2}{|c|}{ Sample size } & \multicolumn{2}{|c|}{ Gender (M/F) } & \multicolumn{2}{|c|}{ Age (years) } & \multirow[t]{2}{*}{ Method } & \multirow[t]{2}{*}{ NOS score } \\
\hline & & & Case & Control & Case & Control & Case & Control & & \\
\hline Hou (2014) & Asian & Small & 50 & 20 & $42 / 8$ & $17 / 3$ & $32.1 \pm 10.0$ & $34.30 \pm 10.13$ & ELISA & 7 \\
\hline Sha (2013) & Asian & Small & 49 & 18 & $35 / 14$ & $11 / 7$ & $31.6 \pm 7.0$ & - & ELISA & 7 \\
\hline Dhir (2013) & Asian & Large & 85 & 20 & $82 / 3$ & - & $33.0 \pm 10.0$ & - & ELISA & 8 \\
\hline $\mathrm{Ma}(2012)$ & Asian & Small & 38 & 38 & $22 / 16$ & $21 / 17$ & $47.4 \pm 1.3$ & $47.5 \pm 1.4$ & ELISA & 7 \\
\hline Soliman (2012) & African & Small & 30 & 10 & $27 / 3$ & - & $29.1 \pm 9.8$ & - & ELISA & 6 \\
\hline Shen (2011) & Asian & Large & 175 & 95 & $146 / 29$ & $83 / 12$ & $29.0 \pm 12.0$ & 30.5 & ELISA & 8 \\
\hline Wang (2010) & Asian & Small & 48 & 30 & $30 / 18$ & $17 / 13$ & $32.5 \pm 11.2$ & $35.8 \pm 16.1$ & ELISA & 7 \\
\hline Sun (2008) & Asian & Large & 62 & 45 & $45 / 17$ & $29 / 16$ & $30.0 \pm 12.0$ & $32.0 \pm 16.0$ & ELISA & 8 \\
\hline Wendling (2008) & Caucasian & Small & 23 & 21 & $18 / 5$ & - & $39.9 \pm 2.1$ & $41.2 \pm 2.7$ & ELISA & 6 \\
\hline Lin (2008) & Asian & Small & 45 & 31 & $32 / 13$ & $13 / 18$ & $20-49$ & $26-46$ & ELISA & 7 \\
\hline de Seny (2008) & Caucasian & Small & 19 & 66 & $12 / 24$ & $14 / 5$ & $41(21-80)$ & $57(21-77)$ & ELISA & 6 \\
\hline Chen (2006) & Asian & Small & 42 & 20 & $34 / 8$ & & $36.6 \pm 12.0$ & - & ELISA & 6 \\
\hline Yang (2004) & Asian & Small & 41 & 28 & $32 / 9$ & $14 / 14$ & $12-42$ & $15-49$ & ELISA & 7 \\
\hline
\end{tabular}

$\mathrm{M}=$ male; $\mathrm{F}$ = Female; ELISA = enzyme linked immunosorbent assay; NOS = Newcastle-Ottawa Scale. 


\section{Meta-analysis of MMP-3 levels and AS risk}

In the meta-analysis, the relationship between MMP-3 level and AS risk was assessed in the random effect model for the observed heterogeneity (cases vs controls: $P=90.9 \%, P<$ 0.001). Results showed that AS cases presented a significantly higher serum MMP-3_level than the controls, based on the SMDs from combined results of all included studies (cases vs controls: SMD $=1.31,95 \% \mathrm{Cl}=0.84-1.78, \mathrm{P}<0.001)$, suggesting that higher serum MMP-3 levels were correlated with increased AS risk (Figure 2).

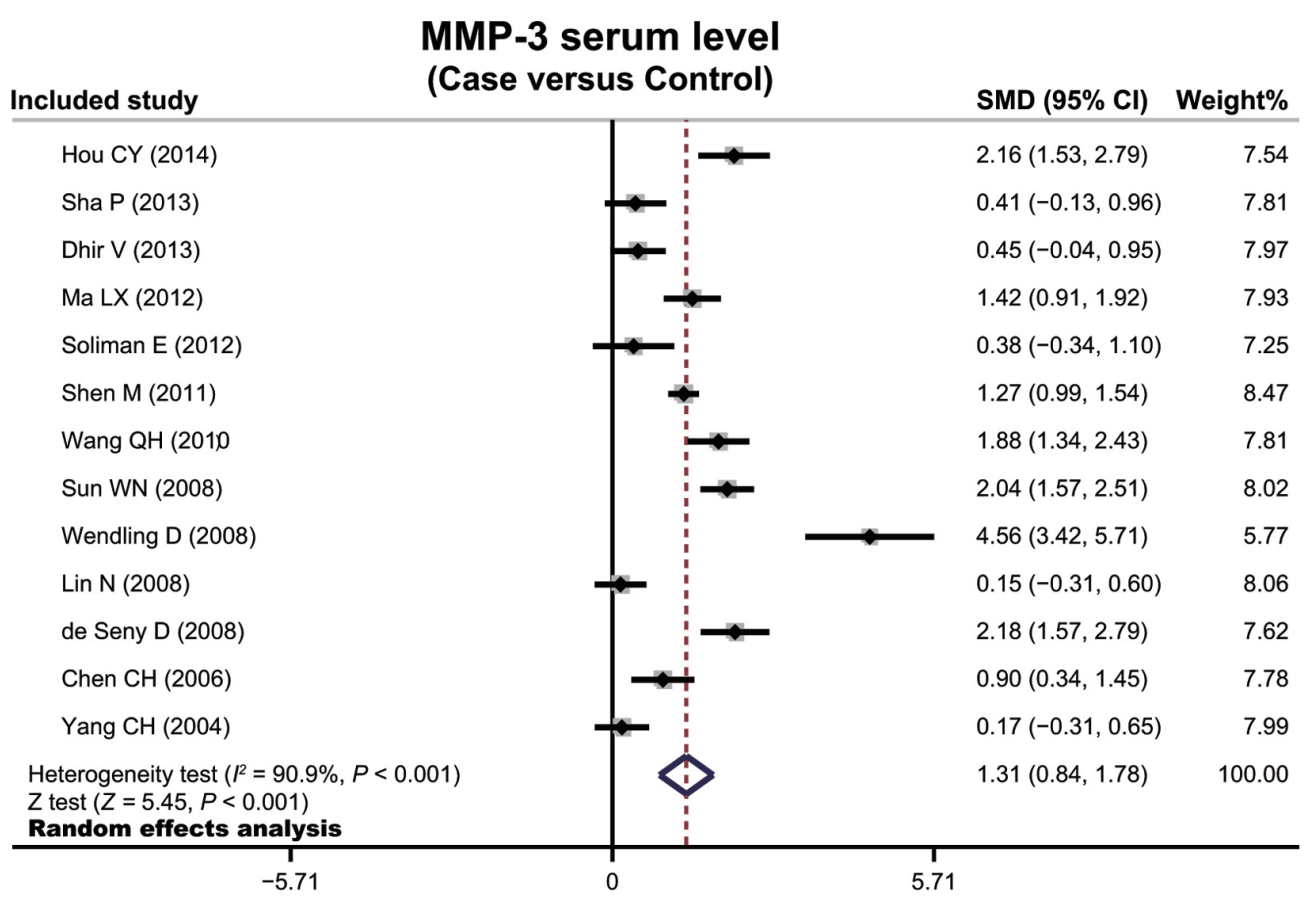

Figure 2. Forest plots for the differences in serum MMP-3 level between patients with ankylosing spondylitis and healthy subjects.

\section{Subgroup analysis}

Since an obvious heterogeneity was found, the differences in MMP-3 levels in patients with AS and the healthy controls were evaluated for subgroups of different explanatory variables. We suggested that an increase in serum MMP-3 level occurred more frequently in patients with AS in comparison to the controls in the Asian and Caucasian subgroups ( $P<0.05$ for both), but not in the African subgroup $(P=0.305)$. In addition, we found positive associations between higher MMP-3 level and AS susceptibility in small sample size studies $(\mathrm{SMD}=1.35,95 \% \mathrm{Cl}=0.72-1.99, \mathrm{P}$ $<0.001)$ as well as in studies with large sample size $(\mathrm{SMD}=1.26,95 \% \mathrm{Cl}=0.50-2.01, \mathrm{P}<0.001)$ (Figure 3). 
MMP-3 serum level

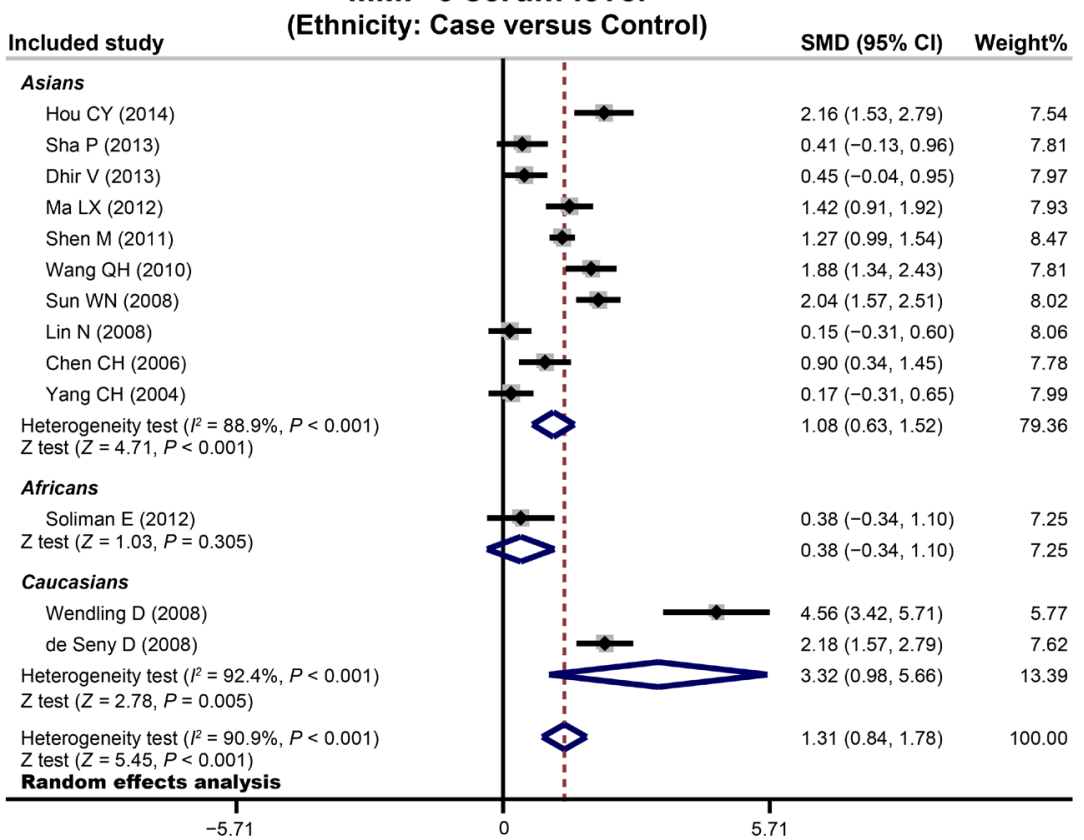

MMP-3 serum level

(Sample: Case versus Control)
Included study

Figure 3. Subgroup analysis for the differences in serum MMP-3 level between patients with ankylosing spondylitis and healthy subjects. 


\section{Sensitive analysis and publication bias}

A sensitivity analysis was performed and the results demonstrated that no single study had the weight to impact on the overall estimate of the association between MMP-3 level and AS risk (Figure 4). Furthermore, we did not observe any obvious asymmetry from the shapes of the funnel plots, which appeared symmetric. In addition, the Egger's regression test suggested the absence of publication bias. Thus, no significant publication bias was detected in the association between MMP-3 level and AS risk $(t=-0.88, P=0.398)$ in our systematic review (Figure 5 ).

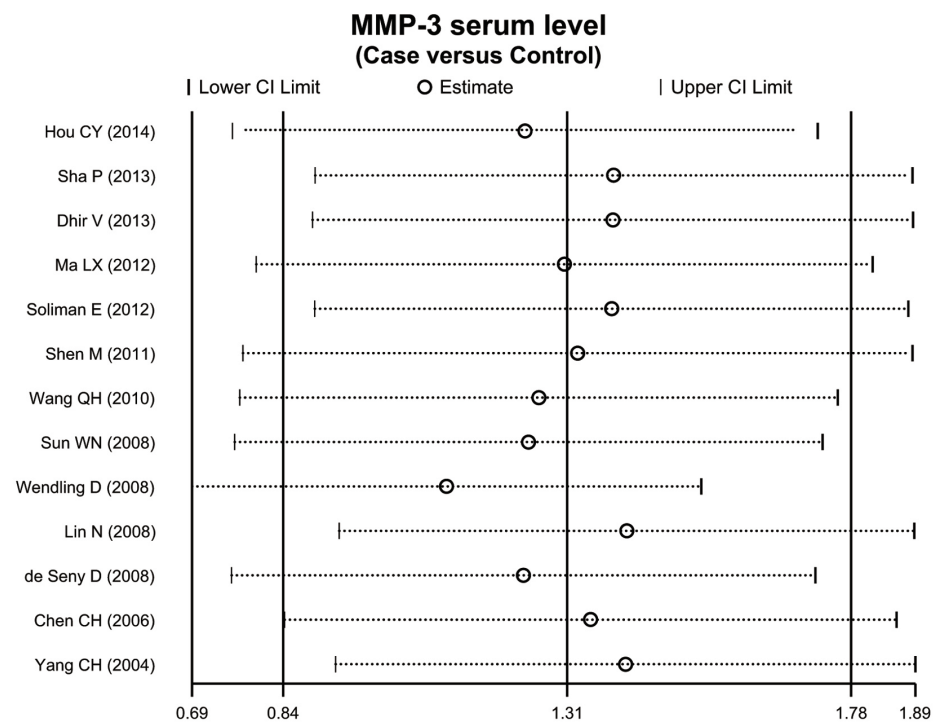

Figure 4. Sensitivity analysis of the summary odd ratio coefficients on the differences in serum MMP-3 level between patients withankylosing spondylitis and healthy subjects.

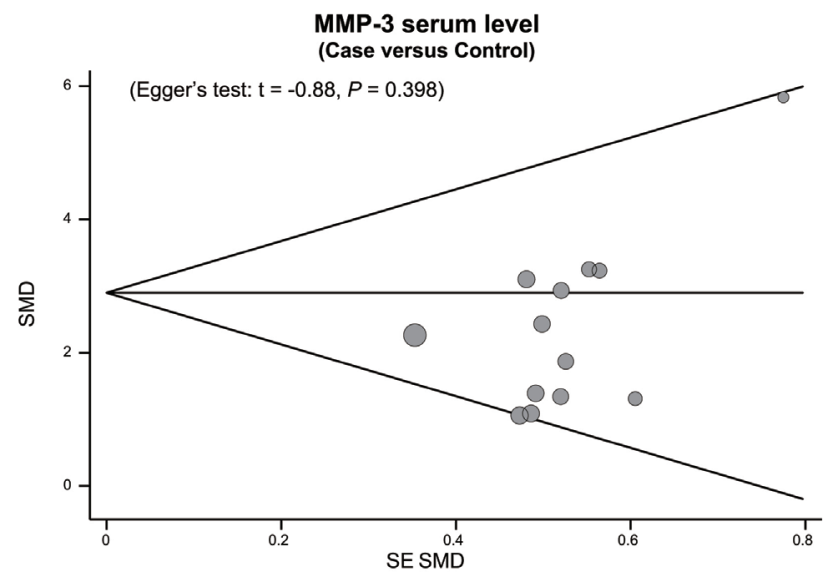

Figure 5. Funnel plot of publication biases on the differences in serum MMP-3 level between patients with ankylosing spondylitis and healthy subjects. 


\section{DISCUSSION}

The present meta-analysis summarizes previous results from relevant studies intending to investigate the relationship of serum levels of MMP-3 with the occurrence and development of AS. The principal results of this meta-analysis revealed that serum MMP-3 levels in patients with AS were apparently higher when compared with those of healthy subjects, indicating that elevated serum levels of MMP-3 might be an important risk factor for the occurrence and progression of AS and could be used as an evaluation tool for disease condition and prognosis in patients with AS. Nevertheless, the exact mechanism underlying the effect of MMP-3 on AS progression remains unclear. AS is one type of chronic, progressive systemic rheumatic disease that has effects on sacroiliac joints, axial skeleton, peripheral joints, and some other extra-articular organs as well as on the cardiovascular system (Gouveia et al., 2012). Patients with AS with abnormal bone density often suffer from osteopenia and osteoporosis, which may play an important role in the pathological changes observed in patients with AS (Magrey and Khan, 2010). Furthermore, AS is an inflammatory disease characterized by inflammation within the sacroiliac joints or axial skeleton. Thus, it is very crucial for patients with AS to prevent the occurrence of inflammatory bone destruction (Bleil et al., 2014). On the other hand, MMP-3 is regarded as one of the most important proteases in the human body, which is involved in the degradation of cartilage matrix (Murphy and Nagase, 2008). MMP-3 is a protease that is mainly secreted by fibroblasts, synovial cells, and cartilage cells. It can degrade various fibronectins and collagens, which are regarded as proteins found in bone and cartilage, such as collagen type IV, VI, and VII (Dhir et al., 2013). MMP3 is implicated in the degradation of the ECM and tissue remodeling in normal biological processes, including embryonic development and cell reproduction as well as in pathological processes such as tumor metastasis and arthritis (Ribbens et al., 2002; Arends et al., 2011). In addition, MMP-3 is expressed in the serum and joint fluid of patients with AS and its level is closely related to the disease activity (Yang et al., 2004a). In our study, a positive association between MMP-3 serum levels and the progression and prognosis of AS was determined. In agreement with our study, De Seny et al. (2008) revealed that MMP-3 serum level apparently increased in patients with AS when compared to healthy subjects and the serum levels of MMP-3 combined with C-reactive protein (CRP) can also reflect the disease activity of AS. In contrast, Wendling et al. (2008) indicated that serum MMP-3 level could not be a serum marker for the progression of AS.

In addition, we also carefully performed stratified analyses based upon ethnicity and sample size to evaluate the correlation between MMP-3 serum level and AS. The ethnicity-stratified analysis results suggested that serum MMP-3 level was closely related to the development and progress of AS among Asians and Caucasians, but not in Africans. These results implied that ethnicity differences, which were caused by the interaction of different geographical environment and genetic differences, may affect this outcome and may be the potential heterogeneity source. In this regard, the highly increased level of serum MMP-3 in patients with AS was associated with the progression of AS. It may reflect joint damage and disease activity and could act as a serologic marker for the improvement or progression of AS.

Our study presents several limitations. First, only three studies out of the 13 included studies present a large sample size of patients, rising concerns regarding the external validity effects. Hence, results from the current meta-analysis need to be confirmed by larger sample size designed studies. Secondly, as shown in the baseline characteristics, four studies did not present the gender information in the controls and four studies failed to get the age information in controls. This may have a restricted 
influence on the heterogeneity test. Third, only serum MMP-3 levels were examined in the current meta-analysis, which may weaken our viewpoints. Hence, additional inflammatory biomarkers should be examined in the same samples to determine which biomarker may be predictive of early damage in AS. Finally, considering that this is a typical cross-sectional epidemiological study, no final conclusion with respect to causality and temporality of the explored connection can be drawn. Thus, all of the above used information could cause an inconsistent outcome.

In summary, this is the first study demonstrating that a serologic biomarker, MMP-3, is a suitable predictor of the progression in AS. Its value as a diagnostic and prognostic maker may be enhanced in future investigations by recruiting a larger case-control population in order to determine whether serum MMP-3 levels change during the development of AS.

\section{Conflicts of interest}

The authors declare no conflict of interest.

\section{REFERENCES}

Arends S, van der Veer E, Groen H, Houtman, PM, et al. (2011). Serum MMP-3 level as a biomarker for monitoring and predicting response to etanercept treatment in ankylosing spondylitis. J. Rheumatol. 38: 1644-1650.

Bakland G and Nossent HC (2013). Epidemiology of spondyloarthritis: a review. Curr. Rheumatol. Rep. 15: 351.

Bleil J, Maier R, Hempfing A, Schlichting U, et al. (2014). Histomorphologic and histomorphometric characteristics of zygapophyseal joint remodeling in ankylosing spondylitis. Arthritis Rheumatol. 66: 1745-1754.

Chen CH, Lin KC, Yu DT, Yang C, et al. (2006). Serum matrix metalloproteinases and tissue inhibitors of metalloproteinases in ankylosing spondylitis: MMP-3 is a reproducibly sensitive and specific biomarker of disease activity. Rheumatology 45: 414-420.

de Seny D, Fillet M, Ribbens C, Maree R, et al. (2008). Monomeric calgranulins measured by SELDI-TOF mass spectrometry and calprotectin measured by ELISA as biomarkers in arthritis. Clin. Chem. 54: 1066-1075.

Dean LE, Jones GT, MacDonald AG, Downham C, et al. (2014). Global prevalence of ankylosing spondylitis. Rheumatology 53: 650-657.

Dhir V, Srivastava R and Aggarwal A (2013). Circulating levels of soluble receptor activator of nf- kappa b ligand and matrix metalloproteinase 3 (and their antagonists) in Asian Indian patients with ankylosing spondylitis. Int. J. Rheumatol. 2013: 814350.

Gouveia EB, Elmann D and Morales MS (2012). Ankylosing spondylitis and uveitis: overview. Rev. Bras. Reumatol. 52: $742-756$.

Halvorsen S, Vollestad NK, Provan SA, Semb AG, et al. (2013). Cardiorespiratory fitness and cardiovascular risk in patients with ankylosing spondylitis: a cross-sectional comparative study. Arthritis Care Res. 65: 969-976.

Higgins JP and Thompson SG (2002). Quantifying heterogeneity in a meta-analysis. Stat. Med. 21: 1539-1558.

Hou CY, Li JQ, Jiang C and Li ZJ (2014). Significance of serum osteopontin and matrix metalloproteinase-3 detection in patients with ankylosing spondylitis. Lab. Med. Clin. 11: 321-322.

Jin L, Weisman M, Zhang G, Ward M, et al. (2005). Lack of association of matrix metalloproteinase 3 (MMP3) genotypes with ankylosing spondylitis susceptibility and severity. Rheumatology 44: 55-60.

Johansson N, Ahonen M and Kahari VM (2000). Matrix metalloproteinases in tumor invasion. Cell Mol. Life Sci. 57: 5-15.

Kassimos DG, Vassilakos J, Magiorkinis G and Garyfallos A (2014). Prevalence and clinical manifestations of ankylosing spondylitis in young Greek males. Clin. Rheumatol. 33: 1303-1306

Lee SH, Lee EJ, Chung SW, Song R, et al. (2013). Renal involvement in ankylosing spondylitis: prevalence, pathology, response to TNF-a blocker. Rheumatol. Int. 33: 1689-1692.

Lin N, Lin X and Deng ZK (2008). The serum matrix metalloproteinase-1,3,9 level in patients with ankylosing spondylitis and its significance. Lab. Med. 23: 357-359.

Ma LX and Yang ZL (2012). The significance of serum matrix metalloproteinase-3 in patients with ankylosing spondylitis. China Health Care Nutr. 22: 311-312.

Magrey M and Khan MA (2010). Osteoporosis in ankylosing spondylitis. Curr. Rheumatol. Rep. 12: 332-336.

Murphy G and Nagase H (2008). Progress in matrix metalloproteinase research. Mol. Aspects Med. 29: 290-308. 
Nagase H, Visse R and Murphy G (2006). Structure and function of matrix metalloproteinases and TIMPs. Cardiovasc. Res. 69: $562-573$.

Peters JL, Sutton AJ, Jones DR, Abrams KR, et al. (2006). Comparison of two methods to detect publication bias in metaanalysis. JAMA 295: 676-680.

Ribbens C, Andre B, Kaye O, Kaiser MJ, et al. (2000). Synovial fluid matrix metalloproteinase-3 levels are increased in inflammatory arthritides whether erosive or not. Rheumatology 39: 1357-1365.

Ribbens C, Martin y Porras M, Franchimont N, Kaiser MJ, et al. (2002). Increased matrix metalloproteinase-3 serum levels in rheumatic diseases: relationship with synovitis and steroid treatment. Ann. Rheum. Dis. 61: 161-166.

Sha P and Lei XP (2013). Expression and significance of HMGB1 and MMP-3 in ankylosing spondylitis J. Shanxi Med. Univ. 44: 933-935.

Shen M, Li XF, Hu XF and Wang XX (2011). The level of serum matrix metalloproteinase-3 and its significance in patients with ankylosing spondylitis. Chin. Remedies Clin. 11: 16-19.

Soliman E, Labib W, el-Tantawi G, Hamimy A, et al. (2012). Role of matrix metalloproteinase-3 (MMP-3) and magnetic resonance imaging of sacroiliitis in assessing disease activity in ankylosing spondylitis. Rheumatol. Int. 32: 1711-1720.

Song F and Gilbody S (1998). Bias in meta-analysis detected by a simple, graphical test. Increase in studies of publication bias coincided with increasing use of meta-analysis. BMJ 316: 471.

Sun NW and Yang HQ (2008). Analysis of Toll-like receptor 4 and matrix metalloproteinase-3 in patients with ankylosing spondylitis. Chin. J. Misdiagn. 8: 2811-2812.

Sun S, Bay-Jensen AC, Karsdal MA, Siebuhr AS, et al. (2014). The active form of MMP-3 is a marker of synovial inflammation and cartilage turnover in inflammatory joint diseases. BMC Musculoskelet. Disord. 15: 93.

van der Linden S, Valkenburg HA and Cats A (1984). Evaluation of diagnostic criteria for ankylosing spondylitis. A proposal for modification of the New York criteria. Arthritis Rheum. 27: 361-368.

Visse R and Nagase H (2003). Matrix metalloproteinases and tissue inhibitors of metalloproteinases: structure, function, and biochemistry. Circ. Res. 92: 827-839.

Wagner C, Visvanathan S, Braun J, van der Heijde D, et al. (2012). Serum markers associated with clinical improvement in patients with ankylosing spondylitis treated with golimumab. Ann. Rheum. Dis. 71: 674-680.

Wang QH, Zhang SZ, Xue J and Wu XH (2010). Serum metalloproteinase-3 levels in assessing efficacy of Etanercept in patients with ankylosing spondylitis. J. Zhejiang Univ. 39: 409-414.

Wei JC, Yen JH, Juo SH, Chen WC, et al. (2011). Association of ORAI1 haplotypes with the risk of HLA-B27 positive ankylosing spondylitis. PLoS One 6: e20426.

Wendling D, Cedoz JP and Racadot E (2008). Serum levels of MMP-3 and cathepsin K in patients with ankylosing spondylitis: effect of TNFalpha antagonist therapy. Joint Bone Spine 75: 559-562.

Yang C, Gu J, Rihl M, Baeten D, et al. (2004a). Serum levels of matrix metalloproteinase 3 and macrophage colony-stimulating factor 1 correlate with disease activity in ankylosing spondylitis. Arthritis Rheum. 51: 691-699.

Yang CH, Huang F, Zhao MS, Gu JR, et al. (2004b). Serum metalloproteinase-3 correlates with disease activitv in ankylosing spondylitis. Chin. J. Rheumatol. 08: 577-582.

Zhang Q, Qi J, Hou S, Du L, et al. (2014). A functional variant of PTPN22 confers risk for Vogt-Koyanagi-Harada syndrome but not for ankylosing spondylitis. PLoS One 9: e96943.

Zintzaras E and loannidis JP (2005a). HEGESMA: genome search meta-analysis and heterogeneity testing. Bioinformatics 21: 3672-3673.

Zintzaras E and loannidis JP (2005b). Heterogeneity testing in meta-analysis of genome searches. Genet. Epidemiol. 28: 123-137. 